\title{
Metformin improves monosodium-iodoacetate- induced osteoarthritis via regulation of pain mediators and autophagy-lysosomal pathway.
}

Hyun Sik Na

Catholic University of Korea School of Medicine

Ji Ye Kwon

Catholic University of Korea School of Medicine

Seung Hoon Lee

Catholic University of Korea School of Medicine

Seon-young Lee

Catholic University of Korea School of Medicine

KyungAh Jung

Catholic University of Korea School of Medicine

Keun Hyung Cho

Catholic University of Korea School of Medicine

Jeong-Won Choi

Catholic University of Korea School of Medicine

Hong-Ki Min

Konkuk University Medical Center

Sung-Hwan Park

Seoul Saint Mary's Hospital

Seok Jung Kim

Uijeongbu Saint Mary's Hospital

MI-LA CHO ( $\square$ iammila@catholic.ac.kr)

Catholic University of Korea

\section{Research article}

Keywords: Osteoarthritis, metformin, pain, cartilage, autophagy, combination therapy

Posted Date: February 10th, 2020

DOI: https://doi.org/10.21203/rs.2.23029/v1 
License: (c) (i) This work is licensed under a Creative Commons Attribution 4.0 International License. Read Full License 


\section{Abstract}

Background Osteoarthritis (OA) is the most common degenerative arthritis associated with pain and cartilage destruction in the elderly. Its pathogenesis involves inflammatory pathways. Metformin is used to treat type 2 diabetes but also exhibits regulation of autophagy pathway. This study investigated the ability of metformin to ameliorate the severity of monosodium iodoacetate (MIA)-induced OA in rats.

Methods Metformin was administered orally every day to rats with OA experimentally induced by intraarticular injection of MIA. Paw-withdrawal latency and threshold were used to assess pain severity. Cartilage damage and pain mediators in dorsal root ganglia were evaluated by histological analysis and a scoring system. Relative mRNA expression was measured by real-time PCR.

Results Metformin ameliorated the progression of experimental OA and exhibited both anti-nociceptive properties and cartilage protection. The metformin-induced down-regulation of pain mediators, including CGRP, in dorsal root ganglia and protection against bone damage were also determined. In chondrocytes from OA patients, metformin decreased expression of genes encoding catabolic factors stimulated by interleukin-1 $\beta$ and inflammatory cell death factors and induced autophagosome-lysosome fusion phenotype. The combination therapy of metformin and celecoxib had significantly less cartilage damage than either the metformin alone group or the control group.

Conclusions These results imply the possibility of therapeutic use of metformin in OA patients, based on its ability to suppress pain and protect cartilage.

\section{Background}

Osteoarthritis (OA) is a chronic and degenerative arthritis and the most common continuous joint disorder in the elderly. The severe pain and irreversible cartilage damage associated with OA reduces the quality of life of affected patients[1]. Thus, treatment of $O A$ is aimed at relieving arthralgia and preventing disease progression, including cartilage degradation, which is irreversible and may aggravate arthritic pain.

However, effective disease-modifying osteoarthritis drugs that prevent cartilage damage in OA are not yet available[2]. While OA has traditionally been considered a non-inflammatory arthritis, recent studies have shown that its pathogenesis includes low-grade inflammation[3] and the involvement of numerous proinflammatory cytokines and matrix metalloproteinases (MMPs) in its aggravation[4]. Targeting of these pro-inflammatory cytokines has thus emerged as a therapeutic option in OA[4]. The destruction of bone and cartilage gives rises to a state of chronic inflammation[5] and is consistent with the finding of a smaller population of differentiated regulatory $T$ (Treg) cells in the synovial membranes of OA vs. RA patients[6]. Interleukin (IL)-10 expression by Treg cells is also reduced in OA patients[7] whereas levels of helper T (Th) 17 cells in their joints are increased[8]. This imbalance of Th17/Treg, and therefore of proand anti-inflammatory cytokines, may contribute to the pathogenesis of OA.

The biguanide metformin has been widely used for the treatment of type 2 diabetes. However, studies of experimental animal models of autoimmune arthritis have shown that metformin also has anti-arthritic 
activity and inhibits bone degradation $[9,10]$. In other reports, metformin restored the balance between Th17 and Treg cells in an animal model of inflammation [11] and decreased both osteoclastogenesis and expression of MMP-9 in vitro[12].

We therefore hypothesized that metformin could ameliorate OA via anti-nociceptive and antiinflammatory effects. Thus, in this study we investigated the therapeutic effect and related mechanism of action of metformin in both an animal model of OA and in chondrocytes from OA patients. The ability of metformin to restore the balance between Th17 and Treg cells and to induce anabolic/catabolic factors was also determined. In addition, the effects of metformin in OA patients were evaluated by monitoring radiographically evaluated disease progression in the knees of diabetic OA patients treated with metformin.

\section{Methods}

\section{Animals}

Seven-week-old male Wistar rats weighing 180 250 g at the start of the experiment were purchased from Central Lab Animal Inc. (Seoul, South Korea). A maximum of three animals per cage were housed in a room with controlled temperature $\left(20-26^{\circ} \mathrm{C}\right)$ and light (12-h light-dark cycle) conditions. The rats had free access to a gamma-ray-sterilized diet (TD 2018S, Harlan Laboratories, Inct / America) and autoclaved $\mathrm{R} / \mathrm{O}$ water. All animal research procedures were conducted in accordance with the Laboratory Animals Welfare Act, the Guide for the Care and Use of Laboratory Animals, and the Guidelines and Policies for Rodent Experiments provided by the Institutional Animal Care and Use Committee (IACUC) of the School of Medicine, The Catholic University of Korea. The IACUC and Department of Laboratory Animals of the Catholic University of Korea, Songeui Campus, accredited the Korean Excellence Animal Laboratory Facility in accordance with the Korean Food and Drug Administration in 2017 and full accreditation by the AAALAC International was acquired in 2018.

\section{Induction of osteoarthritis and treatment with metformin}

Animals were randomly assigned to the treatment or control groups before the study began. After anesthetization with isoflurane, the rats were injected with $3 \mathrm{mg}$ of monosodium iodoacetate (MIA) (Sigma, St. Louis, MO, USA) dissolved in a 50-ml volume, using a $26.5-\mathrm{G}$ needle inserted through the patellar ligament into the intra-articular space of the right knee. Metformin $100 \mathrm{mg} / \mathrm{kg}$ and the NSAID celecoxib $80 \mathrm{mg} / \mathrm{kg}$ were administered orally to the MIA-induced rats. In addition Metformin \& celecoxib complex was administered at a concentration of metformin $50 \mathrm{mg} / \mathrm{kg}$.

\section{Assessment of pain behavior}

Nociception in MIA-treated rats randomized to the different experimental groups was tested using a dynamic plantar aesthesiometer (Ugo Basile, Gemonio, Italy). The device is an automated version of the 
von Frey hair assessment procedure and is used to assess mechanical sensitivity. The assessment was conducted by placing the rats on a metal mesh surface in an acrylic chamber in a temperature-controlled room $\left(20-26^{\circ} \mathrm{C}\right)$, where they rested for $10 \mathrm{~min}$ before the touch stimulator unit was positioned under each animal. An adjustable angled mirror was used to place the stimulating microfilament (0.5-mm diameter) below the plantar surface of the hind paw. When the instrument was activated, a fine plastic monofilament advanced at a constant speed and touched the paw in the proximal metatarsal region. The filament exerted a gradually increasing force on the plantar surface, starting below the threshold of detection and increasing until the stimulus became painful, as indicated by the rat's withdrawal of its paw. The force required to elicit a paw-withdrawal reflex was recorded automatically and measured in $\mathrm{g}$. A maximum force of $50 \mathrm{~g}$ and a ramp speed of $25 \mathrm{~s}$ were used for all aesthesiometer tests.

\section{Assessment of weight bearing}

Weight balance in MIA treated rat was analysed to incapacitance meter (IITC Life Science, CA, USA). The rats are allowed to acclimate for 5 minutes in an acrylic holder. After 5 minutes, fix both feet of the rat to the pad and measure the weight balance for 5 seconds. Repeat the three measurements in the same manner. Determine the weight of the unguided and guided legs and substitute them in the formula to find the\% value. The\% value is obtained by comparing the leg with and without osteoarthritis.

\section{Histopathological analysis}

Knee joints were collected from each group at 2 weeks post-MIA induction. The tissues were fixed in 10\% formalin solution, decalcified using Decalcifying Solution-Lite (Sigma, St. Louis, MO, USA), and embedded in paraffin. Sections of 4- to 5- $\mu \mathrm{m}$ thickness were cut, dewaxed using xylene, dehydrated through an alcohol gradient, and then stained with hematoxylin and eosin (H\&E) and safranin 0 .

\section{Immunohistochemistry}

Paraffin-embedded sections were incubated at $4^{\circ} \mathrm{C}$ with the following primary monoclonal antibodies: anti-transient receptor potential cation channel subfamily V member (TRPV)-1 (R\&D Systems), anticalcitonin gene-related peptide (CGRP), anti-IL-1 $\beta$, anti-IL-17, anti-MMP-3, anti-inducible nitric oxide synthase (iNOS), anti-caspase-1, anti-phospho-MLKL (Abcam, UK), and anti-phospho-AMPK (Cell Signaling Technologies, MA, USA). The samples were then incubated with the respective secondary biotinylated antibodies, followed by a 30-min incubation with a streptavidin-peroxidase complex. The reaction product was developed using 3, 3-diaminobenzidine chromogen (Dako, USA). 


\section{Immunofluorescence}

Human chondrocytes were stained using FITC-conjugated anti-BODIPY (Sigma, St. Louis, MO, USA), PEconjugated anti-LC3b, and APC-conjugated anti-LAMP1 (Santa Cruz Biotechnology, USA).

\section{Real-time polymerase chain reaction (PCR)}

Total RNA isolated from human chondrocytes using the TRIzol reagent (Molecular Research Center, USA) was used to synthesize cDNA. The relative expression of specific mRNA was quantified by real-time PCR using Sensil FAST SYBR (Bioline, USA) and the following sense and antisense primers: for MMP-1, 5'-CTG AAG GTG ATG AAG CAG CC-3' (sense) and 5'-AGT CCA AGA GAA TGG CCG AG-3' (anti-sense); for MMP-3, 5'-CTC ACA GAC CTG ACT CGG TT-3' (sense) and 5'-CAC GCC TGA AGG AAG AGA TG-3' (anti-sense); for MMP-13, 5'-CTA TGG TCC AGG AGA TGA AG-3' (sense) and 5'-AGA GTC TTG CCT GTA TCC TC-3' (antisense); for TIMP1, 5'-AAT TCC GAC CTC GTC ATC AG-3' (sense) and 5'-TGC AGT TTT CCA GCA ATG AG-3' (anti-sense); for TIMP3, 5'-CTG ACA GGT CGC GTC TAT GA-3' (sense) and 5'-GGC GTA GTG TTT GGA CTG GT-3' (anti-sense); for AMPKa1, 5'-AAC TGC AGA GAG CCA TTC ACT TT-3' (sense) and 5'-GGT GAA ACT GAA GAC AAT GTG CTT-3' (anti-sense).

\section{Western blot analysis}

Proteins were separated by SDS-PAGE and transferred onto a nitrocellulose membrane (Amersham Pharmacia Biotech, Piscataway, NJ, USA). Western blotting was performed using a SNAP i.d. protein detection system (Millipore). The hybridized bands were detected using an enhanced chemiluminescence (ECL) detection kit (Thermo Fisher Scientific, MA. USA) and the following antibodies: anti-phosphoAMPK(Cell Signaling Technologies, MA, USA), anti-caspase-1, anti-caspase-3, anti-GAPDH (Abcam, UK), and anti-goat anti-rabbit lgG-HRP.

\section{In vivo micro-computed tomography (CT) imaging and analysis}

Micro-CT imaging and analysis were performed using a bench-top cone-beam type in vivo animal scanner (mCT 35; SCANCO Medical, Br” uttisellen, Switzerland). The animals were imaged at settings of $70 \mathrm{kVp}$ and $141 \mu \mathrm{A}$ using an aluminum $0.5-\mathrm{mm}$ thick filter. The pixel size was $8.0 \mu \mathrm{m}$ and the rotation step was $0.4^{\circ}$. Cross-sectional images were reconstructed using a filtered back-projection algorithm (NRecon software, Bruker micro CT, Belgium). For each scan, a stack of 286 cross-sections was reconstructed at 2,000 × 1,335 pixels. Bone volume and surface were analyzed at the femur. 


\section{Human articular chondrocyte differentiation}

Articular cartilage for human was acquired from patients to Replacement arthroplasty or joint replacement surgery. cartilage obtained from the patient was digested and reacted with $0.5 \mathrm{mg} / \mathrm{ml}$ hyaluronidase, $5 \mathrm{mg} / \mathrm{ml}$ protease type 欧, and $2 \mathrm{mg} / \mathrm{ml}$ collagenase type V. Finally, Chondrocytes were incubated Dulbecco's Modified Eagle Medium (DMEM) including 10\% fetal bovine serum.

\section{$\mathrm{KL}$ grade analysis in OA patients}

The radiographs of both knees of OA patients treated with NSAIDs for $>3$ years were evaluated retrospectively. The data from 60 patients divided into two groups, diabetic patients who had taken metformin to control their blood glucose level and non-diabetic patients, were collected and analyzed (UC18RESI0038).

\section{Statistical analysis}

The results are expressed as the mean \pm standard deviation (SD) and were obtained from three separate experiments. Statistical significance was determined according to the Mann-Whitney U-test or an ANOVA with Bonferroni's post-hoc test, performed using GraphPad Prism (version 5.01, GraphPad Software, San Diego, CA). A p value $<0.05$ was considered to indicate statistical significance.

\section{Results}

\section{Metformin suppresses pain in MIA-induced OA rats.}

The ability of metformin to improve pain in MIA-induced OA rats was assessed based on secondary tactile allodynia. Compared to control MIA rats, metformin administration increased paw-withdrawal latency (PWL), the paw-withdrawal threshold (PWT), and weight bearing, as determined in an automated von Frey hair assessment (Figure 1A, B). In addition, expression of TRPV1 and CGRP in dorsal root ganglia (DRG) was lower in metformin-treated than in control rats. but TRPV1 did not difference (Figure 1C). These results imply that metformin can attenuate OA-related pain by inhibiting nociception and pain severity.

\section{Protective effects of metformin in the knee joints of MIA-induced OA rats}


Quantitative micro-CT showed that metformin protected the femurs of MIA-induced OA rats (Figure 2A), as shown by the increases in bone volume and bone surface in the treated vs. the control group (Figure 2B). Safranin O staining of the femur revealed that metformin decreased cartilage destruction of the knee joint (Figure 2C). Additionally, metformin administration reduced both the OARSI and the total Mankin score compared to the control group (Figure 2D). Together, these data demonstrate that metformin ameliorates $\mathrm{OA}$ progression by suppressing bone and cartilage damage during $\mathrm{OA}$ development.

\section{Metformin reduces the levels of inflammatory mediators and catabolic factors in the synovium of OA rats}

Immunohistochemistry was used to detect inflammatory mediators and the production of catabolic factors in the joints of OA rats. In the metformin-treated group, expression of MMP-3, IL-1ß, iNOS, and IL17 was reduced (Figure $3 \mathrm{~A}$ ). These results imply that metformin limits the catabolic and inflammatory responses in MIA-induced OA rats.

\section{Metformin decreases the catabolic response of OA rat chondrocytes}

Real-time PCR was conducted to evaluate the therapeutic function of metformin in the IL-1 $\beta$-stimulated chondrocytes of OA rats: Expression of the genes encoding MMP-1, -3 and -13 was down-regulated in metformin- vs. vehicle-treated cultures (Figure 3B) whereas TIMP-1 and-3 mRNA levels were increased (Figure 3C). An increase in AMPK, which is activated by metformin and leads to decreased expression of pro-catabolic factors in chondrocytes[13], was also observed (Figure 3D). These results imply that the mechanisms underlying OA prevention by metformin include control of the reciprocal balance between anabolic and catabolic responses and enhanced AMPK activation.

\section{Metformin regulates inflammation-induced cell death in the joints of MIA-induced OA rats}

Immunohistochemistry was also used to detect autophagy and the levels of cell death mediators in MIAtreated rat joints. Expression of AMPK, which is also an activator of autophagy, was significantly increased by metformin administration whereas both caspase- 1 and p-MLKL levels were significantly reduced (Figure 4A). Western blotting revealed increases in the expression of caspase- 3 and AMPK and a decrease in that of caspase-1 in metformin-treated chondrocytes (Figure 4B). These data imply that in a rat model of $O A$, metformin attenuates necrotic cell death via the phosphorylation of AMPK.

\section{Metformin promotes autophagy in human chondrocytes}


Human OA chondrocytes stimulated by IL-1 $\beta$ were examined for autophagolysosome markers by immunofluorescence staining. In the metformin group, the number of LC3B- and -LAMP1 positive cells increased (Figure 5A, B). In addition, patients on combination therapy consisting of metformin and celecoxib had significantly less cartilage damage than either the metformin alone group or the control group (Figure 6A, B). These findings indicate that metformin regulates the expression of autophagolysosome markers in chondrocytes.

\section{Metformin inhibits clinical grade OA and exhibits anti-inflammatory effect in OA patients}

The radiographs of both knees of OA patients treated with NSAIDs for $>3$ years were evaluated retrospectively. The 60 patients were divided into two groups: diabetic patients who had taken metformin to control their blood glucose level and non-diabetic patients. The ability of metformin to inhibit the clinical symptoms of $\mathrm{OA}$ was evaluated based on the $\mathrm{KL}$ grade. The results showed a lower mean $\mathrm{KL}$ grade in the metformin group than in the non-metformin group (Figure 7A).

\section{Discussion}

Although the anti-arthritic and anti-inflammatory effects of metformin have been reported[11, 12], little is known regarding its potential therapeutic effect in OA, neither in an animal model nor in patients. The present study demonstrated the therapeutic effect of metformin in rats with MIA-induced OA and thus its potential clinical use in OA patients. Specifically, we showed that metformin ameliorates experimental OA by suppressing pain mediators and cartilage damage. A previous study recommended the use of antiinflammatory agents in the treatment of OA based on the low-grade chronic inflammatory response associated with the pathogenesis of the disease and the resulting bone deformation[14]. Indeed, blocking IL-6 production prevents OA progression in animal models while NSAIDs suppress inflammatory cytokine levels in OA synovial fluid[15, 16]. Metformin seems to have not only anti-glycemic but also antiinflammatory effects[17]. In the present study, administration of metformin prevented the progression of MIA-induced OA in rats and was associated with a lower $\mathrm{KL}$ grade in the knees of OA patients. The underlying mechanisms included the inhibition of cartilage destruction, the suppression of proinflammatory cytokines and catabolic enzymes, and the amelioration of pain severity.

In the pathogenesis of $\mathrm{OA}$, catabolic activity results in the irreversible degradation of bone and cartilage, which further contributes to the pain level. Catabolic mediators, such as MMPs, have been shown to exacerbate OA severity by promoting cartilage damage[18] whereas TIMPs suppresses MMP activity and maintain cartilage[19,20]. The balance between anabolic and catabolic activities is thought to play an important role in regulating cartilage and tissue repair in OA patients[21]. In addition, metformin, as an agonist of AMPK, induces cartilage maintenance by suppressing inflammation-induced cartilage degradation[13]. A recent study showed smaller Th17 and Treg populations in the peripheral blood of OA 
vs. RA patients[22]. We found that metformin induced activation of AMPK as well as reciprocal regulation of both MMPs/TIMPs and the Th17/Treg population in the chondrocytes and PBMCs of OA patients. These results imply the possibility of using metformin as a disease-modifying OA drug, based on its ability to regulate the immunologic and inflammatory/catabolic pathways. Clinically, OA patients suffer considerable pain, which in addition to severe disabilities results in significant socioeconomic costs[23]. Pro-inflammatory cytokines such as IL- 6 and tumor necrosis factor (TNF)-a have been shown to sensitize nociceptors in rats $[24,25]$ whereas TNF-a blockade attenuated pain behaviors and joint destruction in a mouse model of arthritis[26]. Both TRPV1 and CGRP have been implicated in the pain response[27, 28] while the inhibition of both was shown to improve metabolic health during aging[28]. In the present study, metformin treatment ameliorated pain severity by increasing PWT, PWL, and improved weight bearing in rats with MIA-induced OA. Metformin also decreased expression of TRPV1 and CGRP in the DRG of OA rats. These results imply that metformin relieves pain through the nociceptive pathway in OA.

\section{Conclusions}

This study demonstrated the therapeutic effect of metformin in OA by mechanisms that include cartilage protection and pain relief. Therefore, metformin should be considered as a potential therapeutic candidate for $\mathrm{OA}$.

\section{Abbreviations}

Osteoarthritis

OA; monosodium iodoacetate:MIA; metalloproteinases:MMPs; regulatory T:Treg; Interleukin:IL; helper $\mathrm{T}: T h$; transient receptor potential cation channel subfamily V:TRPV; calcitonin gene-related peptide:CGRP; inducible nitric oxide synthase:iNOS; tumor necrosis factor:TNF; Osteoarthritis Research Society International:OARSI; computed tomography:CT; dorsal root ganglia:DRG

\section{Declarations}

\section{Ethics approval and consent to participate}

Animals

The procedures were approved by the Animal Research Ethics Committee of the Catholic University of Korea, and conformed to the National Institutes of Health of the United States guidelines.

Humans

The procedures were approved by the Research Ethics Committee of Uijeongbu St. Mary's Hospital 


\section{Availability of data and material}

All data are available in the manuscript or upon request to the authors.

\section{Competing interests}

The authors declare that they have no competing interests.

\section{Funding}

This research was supported by the Bio \& Medical Technology Development Program of the National Research Foundation (NRF) funded by the Korean government (MSIT) (NRF-2017M3A9B4028022). Further support was supported by the National Research Foundation of Korea (NRF) grant funded by the Korea government (MSIP) (NRF-2017R1A2B3007688)

\section{Authors' contributions}

HSN, JYK, SHL, SYL, SJK and MLC Conception and design of study; HSN, JYK and SHL Acquisition of data; HSN, JYK, KAJ, KHC and HKM Analysis and interpretation of data; HSN and MLC Drafting the article; HSN and MLC Revising the article critically; MLC and SJK acquired funding.

\section{Acknowledgements}

None.

\section{Authors' information}

${ }^{1}$ The Rheumatism Research Center, Catholic Research Institute of Medical Science, The Catholic University of Korea, College of Medicine, Seoul, South Korea ${ }^{2}$ Divison of Rheumatology, Department of Internal Medicine, The Catholic University of Korea, 137-040, South Korea ${ }^{3}$ Impact Biotech, Korea 505 Banpo-Dong, Seocho-Ku, 137-040, Seoul, Korea ${ }^{4}$ Department of Orthopedic Surgery, Uijeongbu St. Mary's Hospital, College of Medicine, The Catholic University of Korea, Seoul, Korea ${ }^{5}$ Division of Rheumatology, Department of Internal Medicine, Seoul St. Mary's Hospital, College of Medicine, The Catholic University 
of Korea, Seoul, South Korea ${ }^{6}$ Division of rheumatology, Department of Internal Medicine, Konkuk university medical center, Seoul, Republic of Korea

\section{References}

1. Kalman DS, Heimer M, Valdeon A, Schwartz H, Sheldon E: Effect of a natural extract of chicken combs with a high content of hyaluronic acid (Hyal-Joint) on pain relief and quality of life in subjects with knee osteoarthritis: a pilot randomized double-blind placebo-controlled trial. Nutr J 2008, 7:3.

2. Tonge DP, Pearson MJ, Jones SW: The hallmarks of osteoarthritis and the potential to develop personalised disease-modifying pharmacological therapeutics. Osteoarthr Cartil 2014, 22(5):609621.

3. Berenbaum F: Osteoarthritis as an inflammatory disease (osteoarthritis is not osteoarthrosis!). Osteoarthr Cartil 2013, 21(1):16-21.

4. Kapoor M, Martel-Pelletier J, Lajeunesse D, Pelletier JP, Fahmi H: Role of proinflammatory cytokines in the pathophysiology of osteoarthritis. Nat Rev Rheumatol 2011, 7(1):33-42.

5. Sokolove J, Lepus CM: Role of inflammation in the pathogenesis of osteoarthritis: latest findings and interpretations. Ther Adv Musculoskelet Dis 2013, 5(2):77-94.

6. Moradi B, Schnatzer P, Hagmann S, Rosshirt N, Gotterbarm T, Kretzer JP, Thomsen M, Lorenz HM, Zeifang F, Tretter T: CD4(+)CD25(+)/highCD127low/(-) regulatory T cells are enriched in rheumatoid arthritis and osteoarthritis joints-analysis of frequency and phenotype in synovial membrane, synovial fluid and peripheral blood. Arthritis Res Ther 2014, 16(2):R97.

7. Li S, Wan J, Anderson W, Sun H, Zhang H, Peng X, Yu Z, Wang T, Yan X, Smith W: Downregulation of IL-10 secretion by Treg cells in osteoarthritis is associated with a reduction in Tim-3 expression. Biomed Pharmacother 2016, 79:159-165.

8. Li YS, Luo W, Zhu SA, Lei GH: T Cells in Osteoarthritis: Alterations and Beyond. Front Immunol 2017, 8:356.

9. Kim EK, Lee SH, Lee SY, Kim JK, Jhun JY, Na HS, Kim SY, Choi JY, Yang CW, Park SH et al: Metformin ameliorates experimental-obesity-associated autoimmune arthritis by inducing FGF21 expression and brown adipocyte differentiation. Exp Mol Med 2018, 50(1):e432.

10. Ursini F, Russo E, Pellino G, D'Angelo S, Chiaravalloti A, De Sarro G, Manfredini R, De Giorgio R: METFORMIN AND AUTOIMMUNITY: A 'NEW DEAL'OF AN OLD DRUG. Front Immuno/2018, 9:1236.

11. Lee SY, Lee SH, Yang EJ, Kim EK, Kim JK, Shin DY, Cho ML: Metformin Ameliorates Inflammatory Bowel Disease by Suppression of the STAT3 Signaling Pathway and Regulation of the between Th17/Treg Balance. PloS one 2015, 10(9):e0135858. 
12. Son HJ, Lee J, Lee SY, Kim EK, Park MJ, Kim KW, Park SH, Cho ML: Metformin attenuates experimental autoimmune arthritis through reciprocal regulation of Th17/Treg balance and osteoclastogenesis. Mediators Inflamm 2014, 2014:973986.

13. Terkeltaub R, Yang B, Lotz M, Liu-Bryan R: Chondrocyte AMP-activated protein kinase activity suppresses matrix degradation responses to proinflammatory cytokines interleukin-1beta and tumor necrosis factor alpha. Arthritis Rheum 2011, 63(7):1928-1937.

14. Wojdasiewicz P, Poniatowski LA, Szukiewicz D: The role of inflammatory and anti-inflammatory cytokines in the pathogenesis of osteoarthritis. Mediators Inflamm 2014, 2014:561459.

15. Latourte A, Maillet J, Cherifi C, Ea H, Bouaziz W, Funck-Brentano T, Cohen-Solal M, Haÿ E, Richette P: IL-6/STAT3 signalling blockade protects against experimental osteoarthritis in mice. Osteoarthr Cartil, 24:S378.

16. Gallelli L, Galasso O, Falcone D, Southworth S, Greco M, Ventura V, Romualdi P, Corigliano A, Terracciano R, Savino R et al: The effects of nonsteroidal anti-inflammatory drugs on clinical outcomes, synovial fluid cytokine concentration and signal transduction pathways in knee osteoarthritis. A randomized open label trial. Osteoarthr Cartil 2013, 21(9):1400-1408.

17. Cameron AR, Morrison VL, Levin D, Mohan M, Forteath C, Beall C, McNeilly AD, Balfour DJ, Savinko T, Wong AK et al: Anti-Inflammatory Effects of Metformin Irrespective of Diabetes Status. Circ Res 2016, 119(5):652-665.

18. Blasioli DJ, Kaplan DL: The roles of catabolic factors in the development of osteoarthritis. Tissue Eng Part B Rev 2014, 20(4):355-363.

19. Zhang FJ, Yu WB, Luo W, Gao SG, Li YS, Lei GH: Effect of osteopontin on TIMP-1 and TIMP-2 mRNA in chondrocytes of human knee osteoarthritis. Exp Ther Med 2014, 8(2):391-394.

20. Kashiwagi M, Tortorella M, Nagase H, Brew K: TIMP-3 is a potent inhibitor of aggrecanase 1 (ADAMTS4) and aggrecanase 2 (ADAM-TS5). J Biol Chem 2001, 276(16):12501-12504.

21. Mueller MB, Tuan RS: Anabolic/Catabolic balance in pathogenesis of osteoarthritis: identifying molecular targets. PM R 2011, 3(6 Suppl 1):S3-11.

22. Penatti A, Facciotti F, De Matteis R, Larghi P, Paroni M, Murgo A, De Lucia O, Pagani M, Pierannunzii L, Truzzi $M$ et al: Differences in serum and synovial CD4+ T cells and cytokine profiles to stratify patients with inflammatory osteoarthritis and rheumatoid arthritis. Arthritis Res Ther 2017, 19(1):103.

23. Glyn-Jones S, Palmer AJ, Agricola R, Price AJ, Vincent TL, Weinans H, Carr AJ: Osteoarthritis. Lancet 2015, 386(9991):376-387.

24. Richter F, Natura G, Loser S, Schmidt K, Viisanen H, Schaible HG: Tumor necrosis factor causes persistent sensitization of joint nociceptors to mechanical stimuli in rats. Arthritis Rheum 2010, 62(12):3806-3814.

25. Brenn D, Richter F, Schaible HG: Sensitization of unmyelinated sensory fibers of the joint nerve to mechanical stimuli by interleukin- 6 in the rat: an inflammatory mechanism of joint pain. Arthritis Rheum 2007, 56(1):351-359. 
26. Boettger MK, Hensellek S, Richter F, Gajda M, Stockigt R, von Banchet GS, Brauer R, Schaible HG: Antinociceptive effects of tumor necrosis factor alpha neutralization in a rat model of antigeninduced arthritis: evidence of a neuronal target. Arthritis Rheum 2008, 58(8):2368-2378.

27. Del Fiacco M, Quartu M, Boi M, Serra MP, Melis T, Boccaletti R, Shevel E, Cianchetti C: TRPV1, CGRP and SP in scalp arteries of patients suffering from chronic migraine. $J$ Neurol Neurosurg Psychiatry 2015, 86(4):393-397.

28. Riera CE, Huising MO, Follett P, Leblanc M, Halloran J, Van Andel R, de Magalhaes Filho CD, Merkwirth C, Dillin A: TRPV1 pain receptors regulate longevity and metabolism by neuropeptide signaling. Cell 2014, 157(5):1023-1036.

\section{Figures}

A
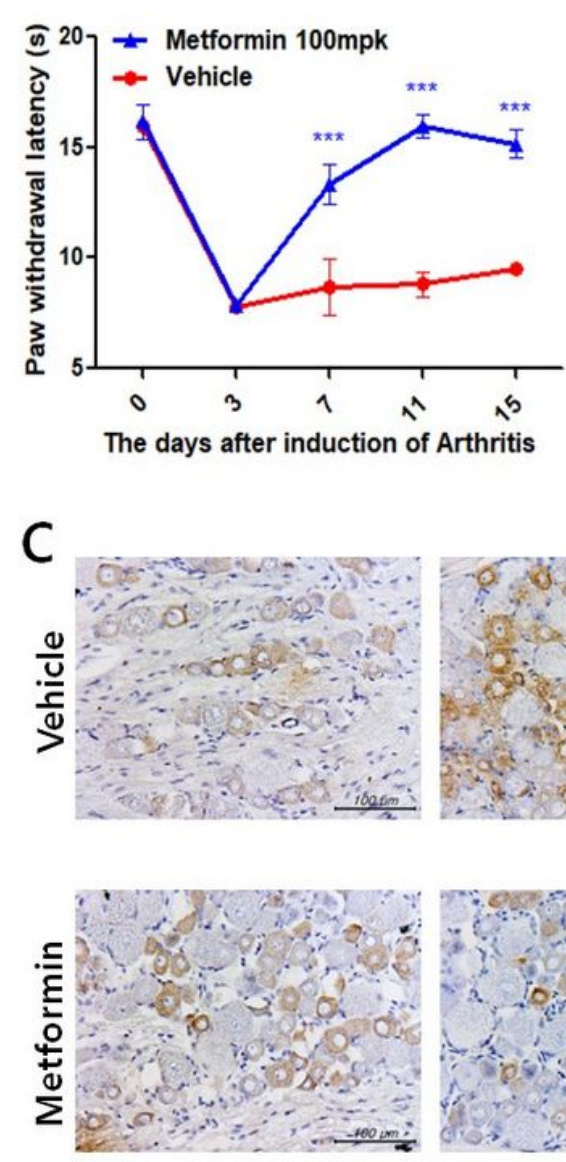

TRPV1
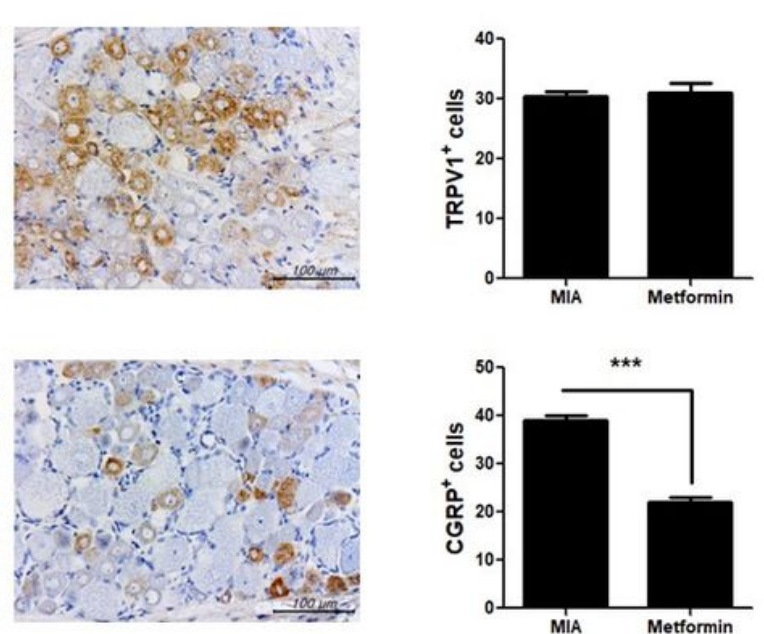

CGRP

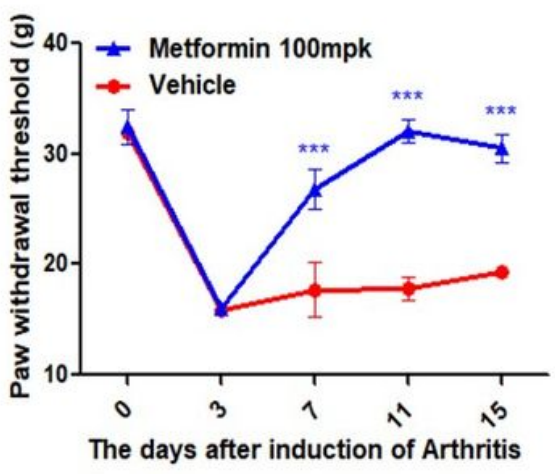

B

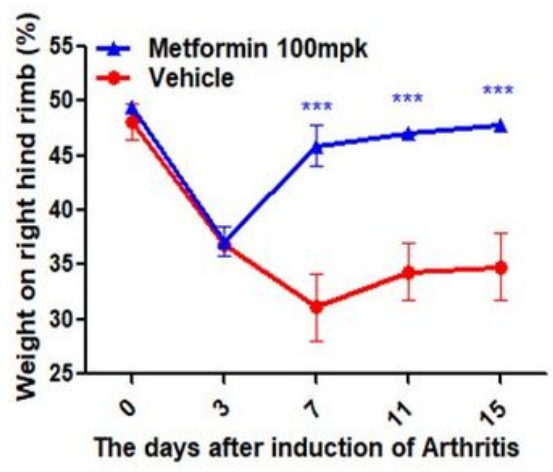

\section{Figure 1}

Metformin reduces pain related to osteoarthritis (OA) (A) Nociceptive testing was performed using a dynamic plantar aesthesiometer (Ugo Basile, Gemonio, Italy), an automated version of the von Frey hair assessment procedure. (B) Weight bearing was assessed using an incapacitance meter (IITC Life 
Science, USA) (C) Dorsal root ganglion tissue from rats in all groups was stained immunohistochemically with specific antibodies to CGRP. (D) Immunohistochemically identified TRPV1- and CGRP-positive cells were counted. The data are reported as the mean \pm SD of three independent experiments in six animals per group. $(* \star * p<0.001)$

A

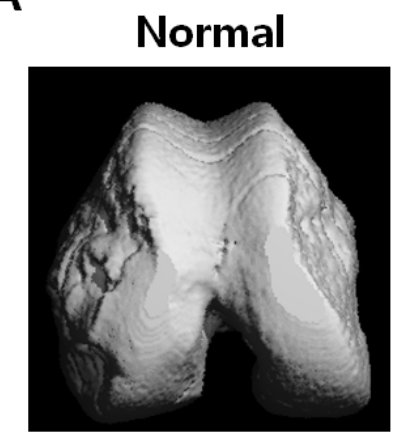

C

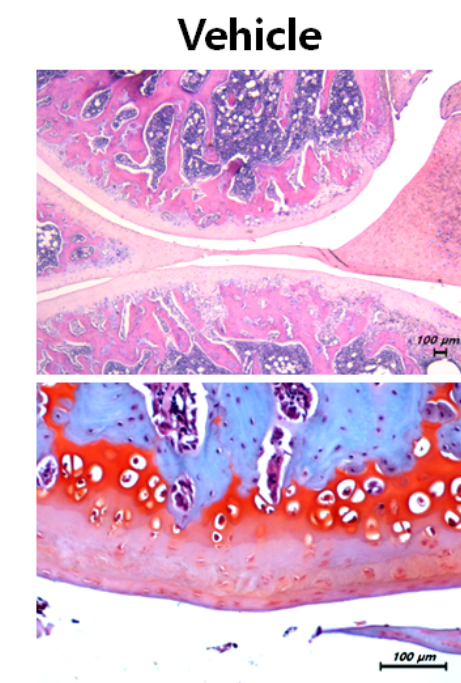

Vehicle

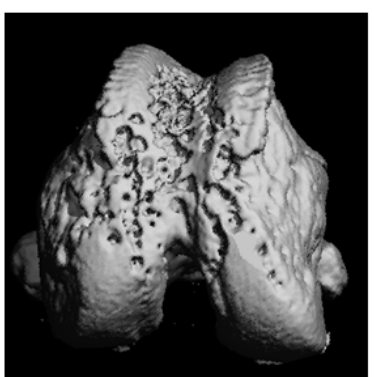

Metformin

\section{Metformin}

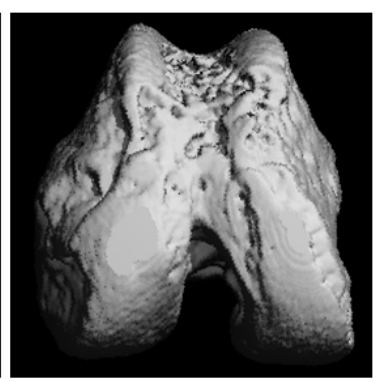

D
B

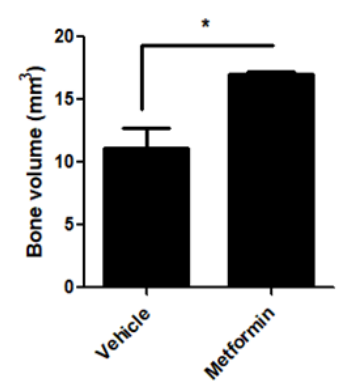

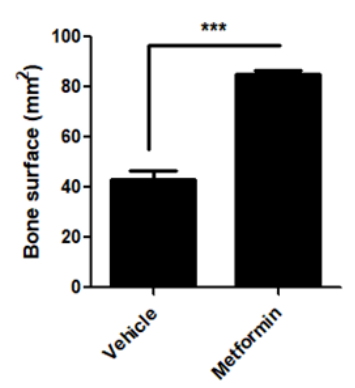
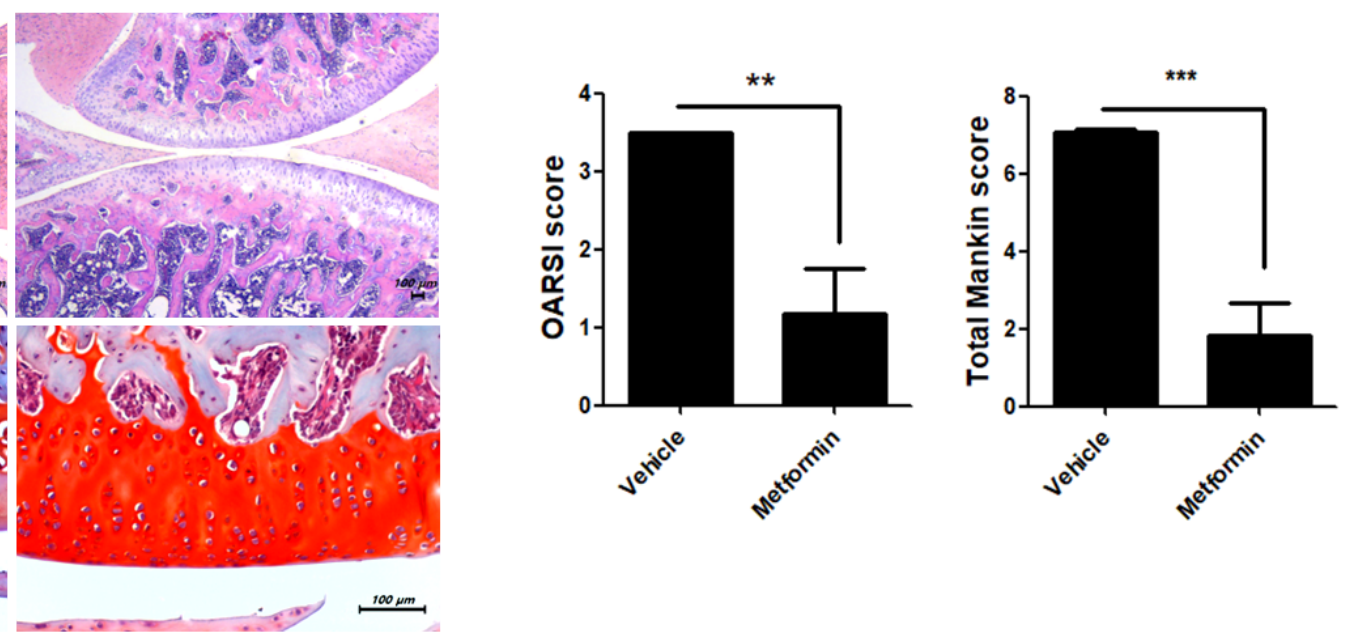

Figure 2

Metformin ameliorates bone and cartilage erosion in OA (A) Twenty cylindrical bone samples were obtained from bone biopsies of 20 dry hemimandibles. The samples of six animals per group were scanned using micro-computed tomography (mCT 35; SCANCO Medical, Wangen-Brüttisellen, Switzerland) (B) Bone volume and bone surface were analyzed using NRecon software (C) Knee-joint tissue samples were acquired from all OA groups at 2 weeks and stained with $\mathrm{H} \& \mathrm{E}$ and safranin $\mathrm{O}(\mathrm{D})$ to determine the Osteoarthritis Research Society International (OARSI) and Mankin scores. The data are reported as the mean \pm SD of three independent experiments. $\left({ }^{*} p<0.05,{ }^{*} p<0.01,{ }^{* \star *} p<0.001\right)$ 
A

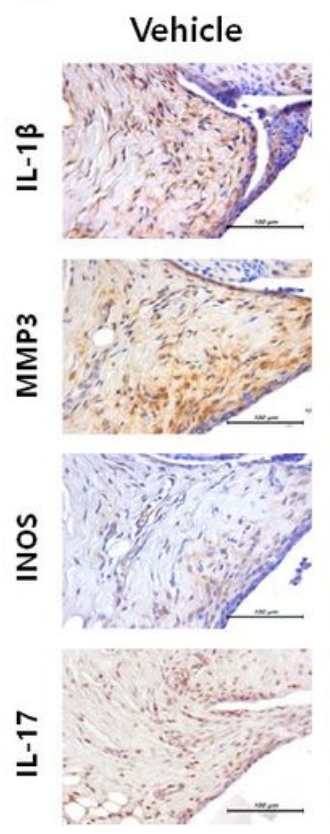

\section{Metformin}
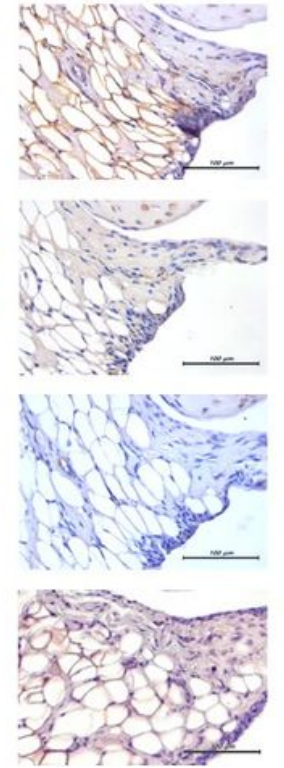
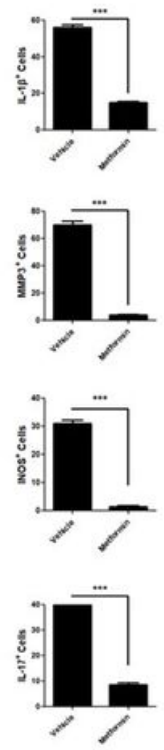

B

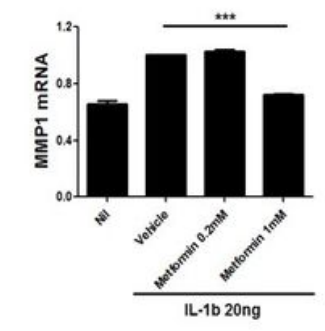

C
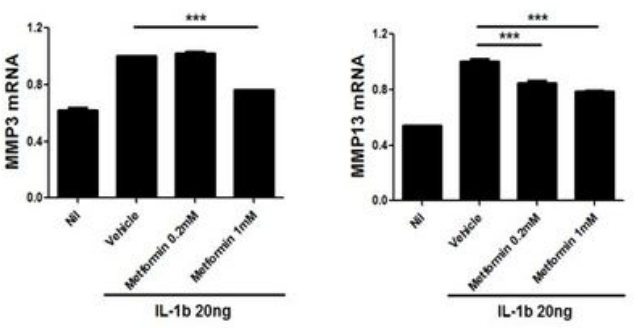

D
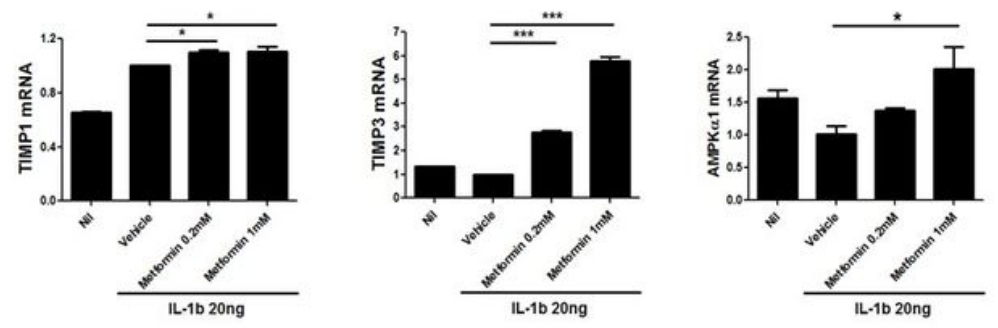

Figure 3

Expression of Inflammatory mediators following metformin administration (A) The expression of IL-1b, MMP3, iNOS, and IL-17 after metformin administration was determined immunohistochemically in the synovium of rats with MIA-induced OA. Positive cells for each antibody are shown on the right. (B) mRNA levels for MMP-1, -3 , and -13, (C) TIMP-1, -3 and (D) AMPKa1 were measured by real time-PCR. The data are reported as the mean \pm SD of three independent experiments in six animals per group. $\left({ }^{*} p<0.05\right.$, ${ }^{*} p<$ $0.01, * \star * p<0.001)$

A
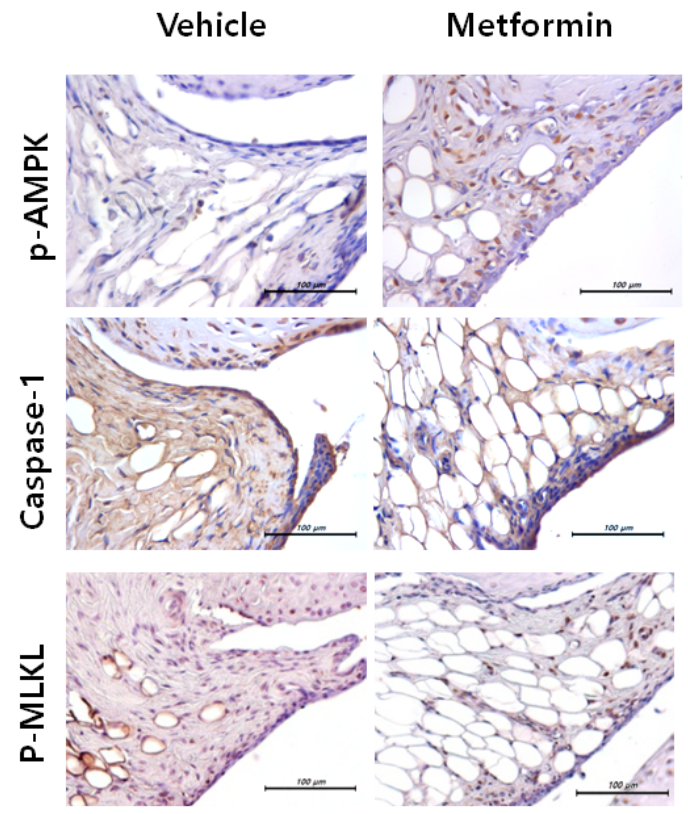

B

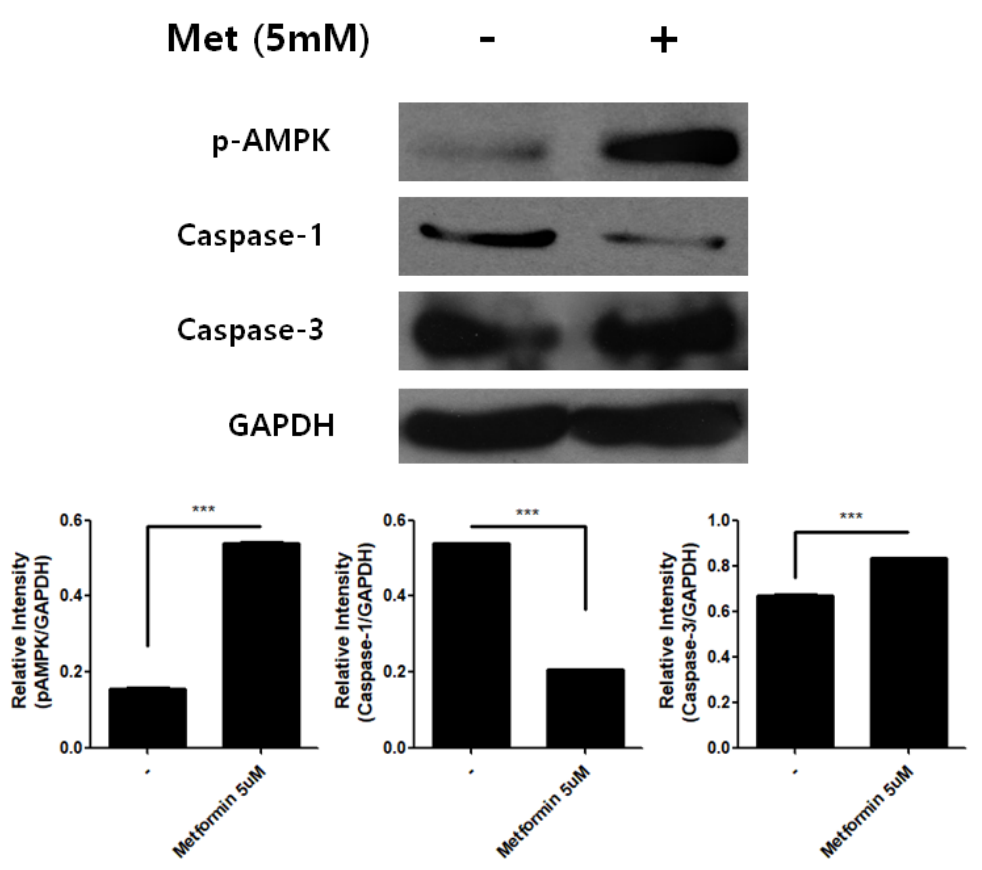




\section{Figure 4}

Expression of cell death mediators following metformin administration (A) The expression of p-AMPK, caspase-1, and p-MLKL in the knee joint tissues of rats with MIA-induced OA rats was determined immunohistochemically. (B) Positive cells for each antibody are shown on the right. (C) Western blot analysis of human chondrocytes treated with metformin. The data are reported as the mean \pm SD of three independent experiments. $(* \star \star p<0.001)$

A

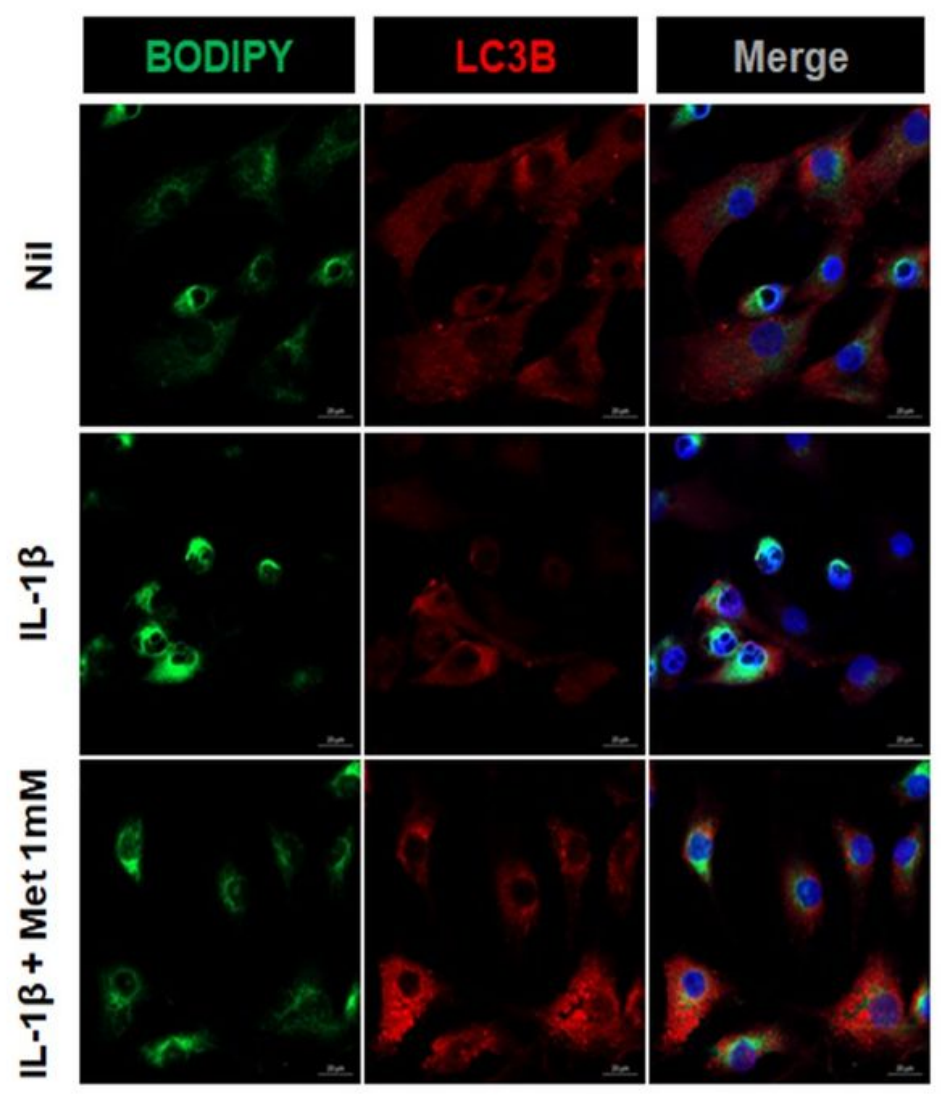

B

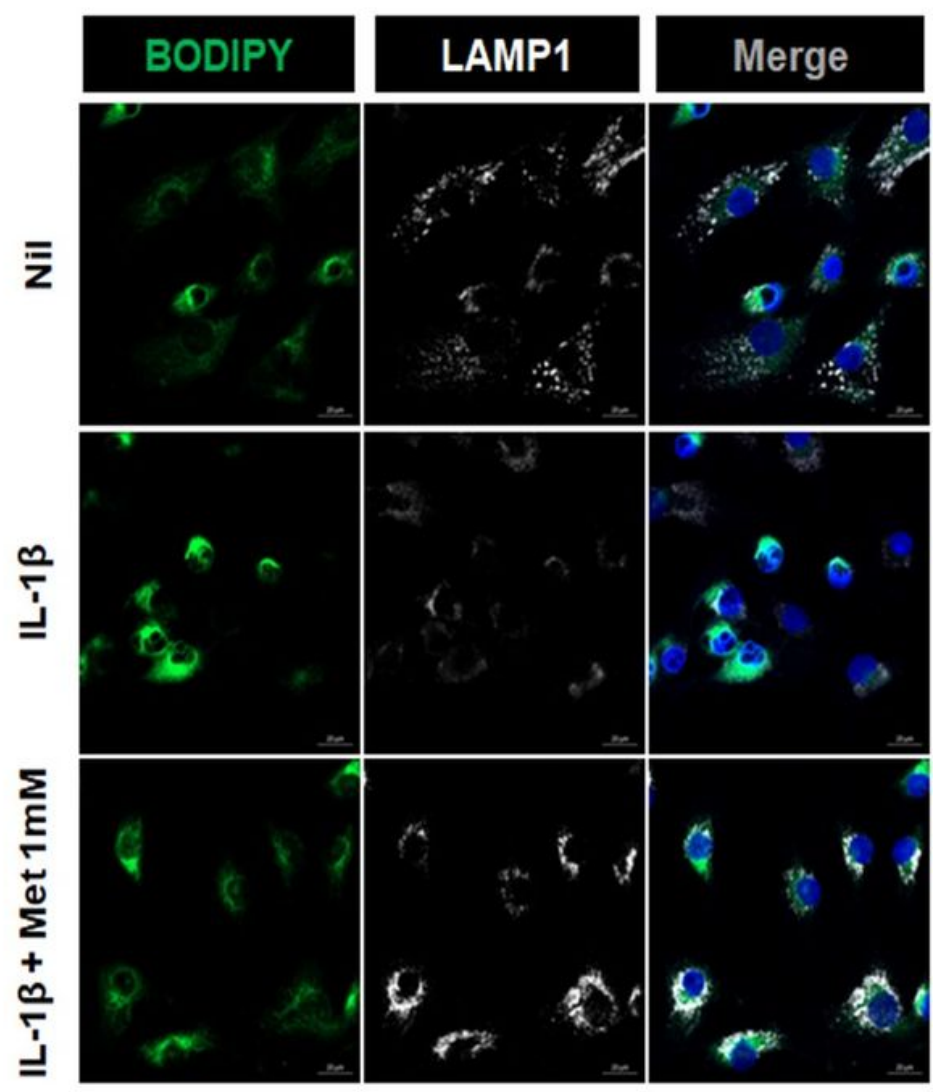

Figure 5

Metformin regulates autophagolysosome via LAMP1 activation. Immunofluorescence was performed to measure the expression of (A) BODIPY, LC3B and (B) LAMP1 in human chondrocytes treated with metformin and IL-1】. 
A

Vehicle

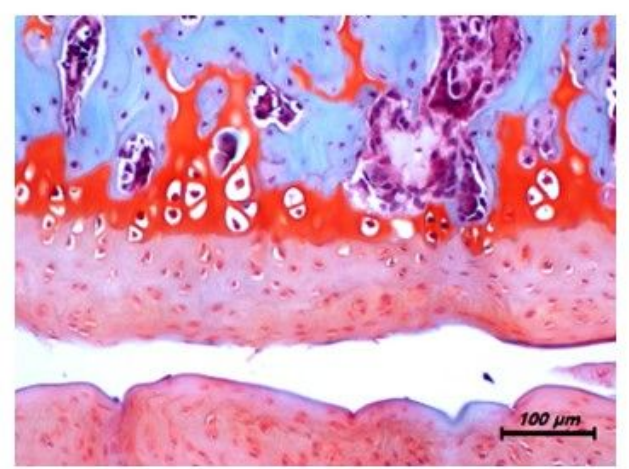

Metformin $50 \mathrm{mg} / \mathrm{kg}$

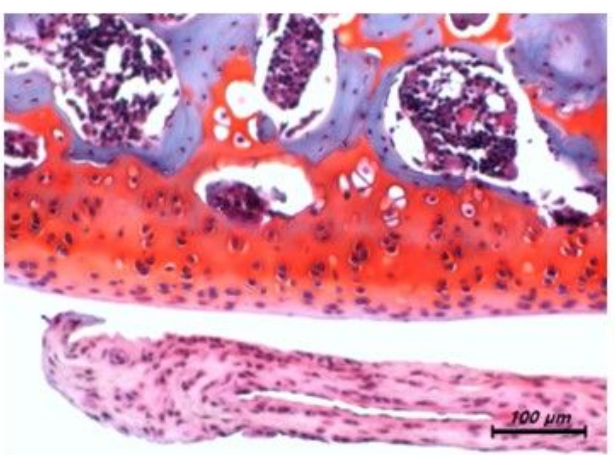

Metformin $50 \mathrm{mg} / \mathrm{kg}$

+ Cele $80 \mathrm{mg} / \mathrm{kg}$

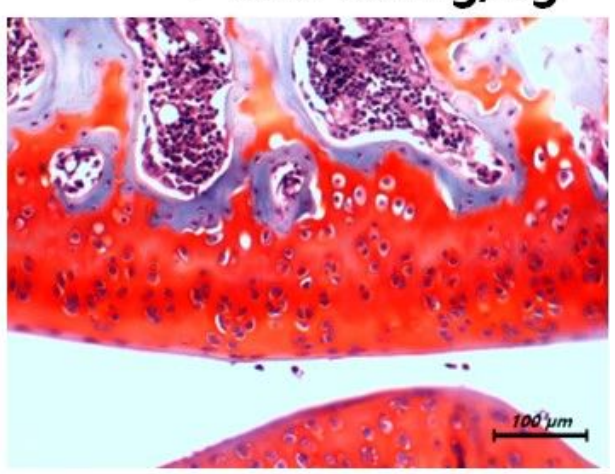

\section{B}
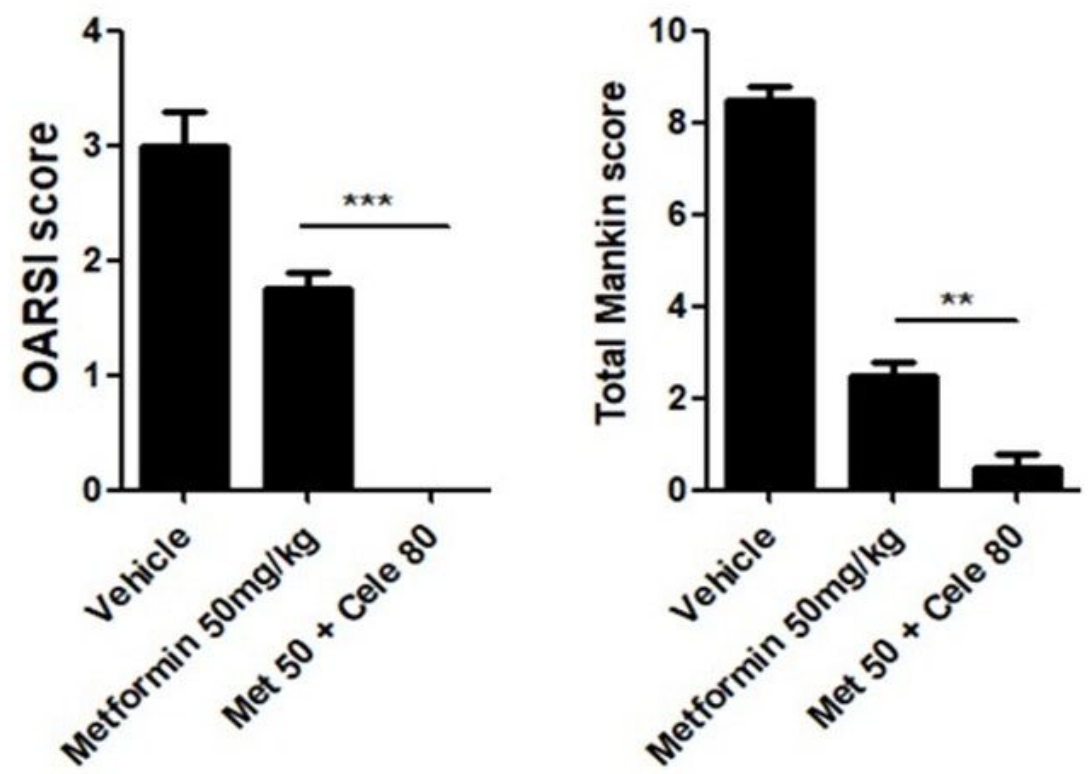

Figure 6

Combined effects of metformin and celecoxib (A) OA was induced in Wistar rats by intra-articular injection of MIA. The rats were then administered metformin and celecoxib in six animals per group. Knee-joint tissue samples acquired from all OA groups at 2 weeks were stained with H\&E and safranin $O$ (B) to evaluate disease severity based on OARSI and Mankin scores. Data are reported as the mean \pm SD of three independent experiments. $\left({ }^{\star *} p<0.01, * \star \star p<0.001\right)$ 
A

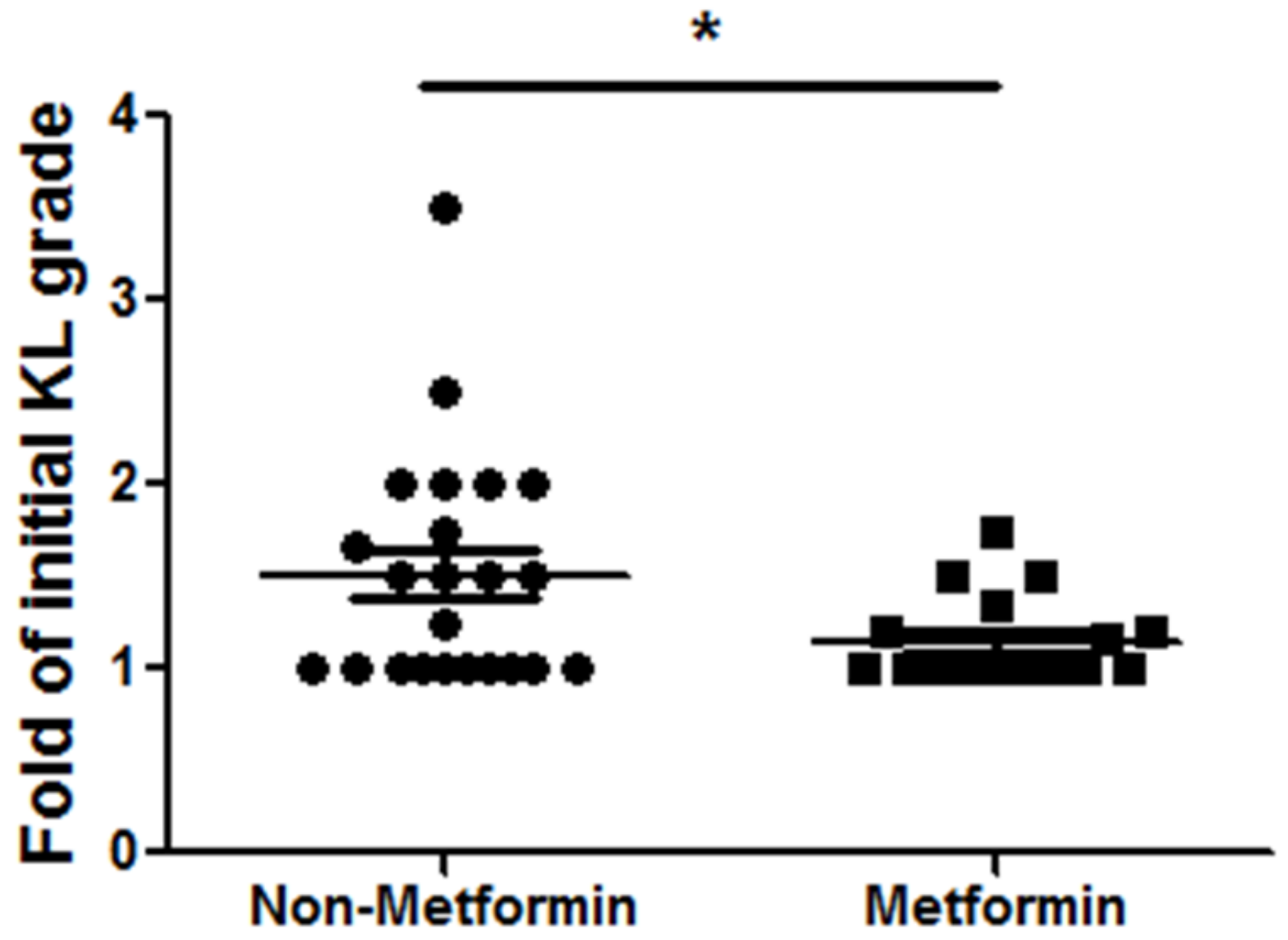

Figure 7

Clinical study of the effect of metformin in OA patients (A) A clinical assessment of OA patients with type 2 diabetes who had been followed-up for $>3$ years showed slower progression of OA in those taking metformin. The OA grade was analyzed based on 3 years of data. $\left({ }^{*} p<0.05\right)$ 